\title{
Genetic analysis of two distinct reproductive strategies in sexual and asexual field populations of an endoparasitic wasp, Venturia canescens
}

\author{
D Li ${ }^{1}, Z_{\text {Zhao }}{ }^{1}$, H Roberts ${ }^{1}$, MV Schneider ${ }^{2}$, U Theopold ${ }^{3}$ and O Schmidt ${ }^{1}$ \\ ${ }^{1}$ Department of Applied and Molecular Ecology, Adelaide University, Glen Osmond SA 5064, Australia; ${ }^{2}$ Institute of Evolutionary and \\ Ecological Sciences, Animal Ecology Section, University of Leiden, P.O. Box 9516, 2300 RA Leiden, The Netherlands; ${ }^{3}$ Department of \\ Molecular Biology and Functional Genomics, Stockholm University, S-10691 Stockholm, Sweden
}

\begin{abstract}
Asexual (thelytokous) females of the parasitoid Venturia canescens, which develop inside another insect, exhibit evolutionarily stable mixtures of life-history strategies, allowing two genetically distinct wasp lines to coexist sympatrically on the same host resources. Since the two asexual lines differ in a virus-like particle protein-coding gene (VLP1), the question is whether the VLP1 gene is genetically associated with the phenotype. The recent isolation of facultative sexual (arrhenotokous) and asexual $V$. canescens strains from the same location in Southern France has enabled an investigation of the genetic basis for the observed phenotypic differences, by comparing the two asexual lines with the corresponding homozygous VLP1 genotypes in arrhenotokous strains. This analysis showed similar patterns of
\end{abstract}

morphological and functional differences exist in the ovaries of the two asexual VLP1 lines and in the two homozygous $V L P 1$ genotypes from the field, suggesting that the VLP1 gene alteration either causes the ovarian phenotype or is genetically closely linked to the putative gene. However, the $V L P 1$-gene may not be the only gene contributing to the phenotypic effects observed in the asexual lines. Although the two VLP1-alleles segregate with the relative differences in the ovary distribution of eggs, the absolute egg numbers differ in the corresponding asexual and sexual genotypes. This suggests that an additional unlinked gene may be involved in the transfer of eggs from the ovarioles into the oviduct.

Heredity (2003) 90, 291-297. doi:10.1038/sj.hdy.6800241

Keywords: reproductive success; egg load; virus-like particle protein; ovary; Venturia canescens; sexual and asexual reproduction

\section{Introduction}

Solitary endoparasitoid wasps that develop inside another insect have evolved special adaptations to manipulate host physiology and to overcome the defence reactions of the host (Schmidt et al, 2001). Superparasitism, where more than one egg is deposited inside the host caterpillar, results in interlarval competition for host dominance, since only one wasp can be supported by the similar-sized host insect. Although it would appear to be advantageous for a female to avoid oviposition of eggs into already parasitised hosts, several experimental studies both in the field and in the laboratory have indicated that parasitic wasps frequently superparasitise (for review, see Van Alphen and Visser, 1990). In fact, it has been shown that superparasitism may not be because of a lack of host discrimination (Salt, 1934, 1961), but can be regarded as an adaptive strategy of parasitoids (Visser et al, 1992; Hubbard et al, 1999).

We have recently identified two genetically distinct laboratory strains of the parthenogenetic endoparasitoid wasp, Venturia canescens, on the basis of a DNA sequence

Correspondence: O Schmidt, Department of Applied and Molecular Ecology, Adelaide University, Glen Osmond SA 5064, Australia.

E-mail: otto.schmidt@adelaide.edu.au

Received 18 June 2002; accepted 11 December 2002 variation of a gene encoding a virus-like particle protein VLP1 (formally p40, Hellers et al, 1996). The two strains differ in a short tandem repeat sequence within the coding region of the VLP1-gene with one allelic wasp strain designated as 'repeat plus' (RP) and the other as 'repeat minus' (RM). Since both allelic genes retain an open reading frame in the VLP1 coding region, two distinct proteins are produced in the two strains, which can be used as genetic markers and allows individuals from the two strains to be distinguished using DNA and protein identification methods (Hellers et al, 1996). The two strains differ in ovary morphology, mainly in the calyx tissue, where the VLP1-gene is expressed (Beck et al, 1999). RM-calyx tissues are less compact and show viruslike particles (VLPs) in the calyx lumen, which are frequently surrounded by unusual membrane material (Beck et al, 2001). This may be an impediment to the passage of eggs from ovarioles into the oviduct with the result that RM-wasps have fewer eggs in their reservoir (Beck et al, 2001). In addition, whereas RM-females produced fewer offspring than RP-females under intrastrain superparasitism, RM-wasps were more successful than RP-wasps under interstrain superparasitism (Beck et al, 1999).

Are these differences in reproductive success related to the ovarian phenotype? Several observations suggest that different egg deposition strategies under conditions 
that favour superparasitism may exploit the dynamics and age distribution of competing larvae. For example, under one-to-one competition the younger wasp larva was shown to have an equal chance of winning a contest for host supremacy for age differences up to $48 \mathrm{~h}$ (Marris and Casperd, 1994, Sirot, 1996), with most fights being determined within $12 \mathrm{~h}$ of hatching (Fisher, 1961, 1963). Thus, if a host is parasitised multiple times over a 24/48$\mathrm{h}$ interval, a larva emerging at a later stage will have a higher probability of being the final survivor, as it will need to win fewer one-to-one fights due to the earlier competitors having largely eliminated each other. The timing of egg deposition may thus be an important factor in determining the outcome of interlarval host competition. Experiments have shown that following host encounters, RM-wasps lay fewer eggs initially and more eggs later compared to RP-wasps (Beck et al, 2001). The observed differences in egg deposition strategies may be because of a lower egg load in RM-wasps (Beck et al, 1999), which correlates with lower egg deposition rates (Trudeau and Gordon, 1989).

Thus, the rate of reproductive success associated with short and long periods of parasitism may reveal functional differences between the two strains. Although the details of competitive events inside the superparasitised caterpillar are not clear, the difference in reproductive success during short and long egg laying periods may be used as an additional phenotypic marker reflecting functional differences between the two strains.

A basic question is whether the VLP1-gene locus is genetically linked to the calyx phenotype. As the genotype in asexual females is fixed, linkage studies require sexually reproducing wasps. The discovery of sexual $V$. canescens strains, containing the two alleles of the VLP1-gene, coexisting with asexual strains in Southern France (Malmberg et al, 2000; Schneider et al, 2002), allowed us to address the questions of whether the VLP1-gene is genetically linked to the phenotype and whether sexual and asexual wasps from field populations differ in reproductive strategies.

Here we report that asexual RM and RP populations from the field show similar differences in ovary morphology and reproductive success to those reported for laboratory RM and RP strains (Beck et al, 1999). Comparison of homozygous RM and RP offspring from sexual strains also show the same pattern of differences suggesting that the VLP1-gene is genetically linked to the phenotype. However, the VLP1-gene may not be the only gene contributing to the phenotypic effects observed in the asexual lines. Although the two VLP1-alleles segregate with the relative differences in the ovary distribution of eggs, the absolute egg numbers differ in the corresponding asexual and sexual genotype. This suggests that an additional unlinked gene may be involved in the transfer of eggs from the ovarioles into the oviduct.

\section{Material and methods}

\section{Insect cultures}

$V$. canescens wasps were reared on final instar caterpillars from Ephestia kuehniella. The insects were maintained in the laboratory at $25^{\circ} \mathrm{C}$ under a $14: 10 \mathrm{~h}$ (light:dark) photoperiod. E. kuehniella caterpillars were fed on bran:wheat germ:brewers yeast (10:2:1) and $V$. canescens adult wasps on a $50 \%$ honey-water solution. To distinguish the larval stages of host and parasitoid, we use the term caterpillar for E. kuehniella, and larva for $V$. canescens larval stages.

\section{Establishment of clonal wasp lines}

$V$. canescens contains nucleic acid-free VLPs, which are produced mainly in the calyx tissue between the ovary and oviduct. One of the four VLP-genes vlp1 exists in two allelic forms, producing two VLP1 proteins. The original laboratory colony from which the first RM and RP lines were derived (Beck et al, 1999, 2001) was founded from material donated by George Salt in the 1960s. These lines are described as RM(thL) (for RM, thelytokous, laboratory) and RP(thL).

A further three pairs of RM and RP lines were established from field-collected specimens. One pair of lines was derived from asexual females collected from two locations near Mildura (Australia) and were used for phenotypic comparison with the laboratory lines. For these lines we use the terms RM(thM), (for RM, thelytokous, Mildura) and RP(thM). The other two pairs of lines were established from sexual and asexual populations recovered from Mont Boron in Southern France (Malmberg et al, 2000; Schneider et al, 2002) and were used for a comparison of both the phenotypes and reproductive success. The asexual genotypes were named $\mathrm{RM}(\mathrm{thB})$ and $\mathrm{RP}(\mathrm{thB})$ and the sexual genotypes were named RM(arB) (for arrhenotokous, Mont Boron) and $\mathrm{RP}(\mathrm{arB})$.

The two alleles constitute a balanced polymorphism, since the two alleles were present in equal proportions in all sexual populations analysed so far. To obtain homozygous RM and RP offspring from the arrhenotokous field population, we set up genetic crosses starting from wasp populations that were mainly heterozygous for the VLP1-gene. Nonmated female wasps were individually paired with single male wasps and kept separately with host caterpillars. After egg deposition the genotypes of the VLP1-gene were determined in both parents using PCR (Figure 1). Those crosses, where both parents were homozygous for the VLP1-gene were used to establish independent homozygous lines. A total of $24 \mathrm{RM}(\operatorname{arB})$ and seven $\mathrm{RP}(\operatorname{arB})$ lines were established and checked for the homozygous RM and RP status in subsequent generations, using random offspring checks. In the following text, we use the term 'strain' to distinguish between the field populations and the term line to distinguish between the two VLP1 genotypes.

$V$. canescens has single locus complementary sex determination (sl-CSD), where heterozygous individuals are female and hemizygous (either haploid or homozygous diploid) individuals are male (Beukeboom, 2001). Under sl-CSD, diploid males, often the result of inbreeding, are typically sterile (Stouthammer et al, 1992; Agoze et al, 1994). As a result of this, it is impractical to maintain isofemale arrhenotokous lines. Therefore, after the homozygous RM and RP status of the independent lines had been verified, the individuals were pooled to create a single pair of lines homozygous for the VLP1 gene. 


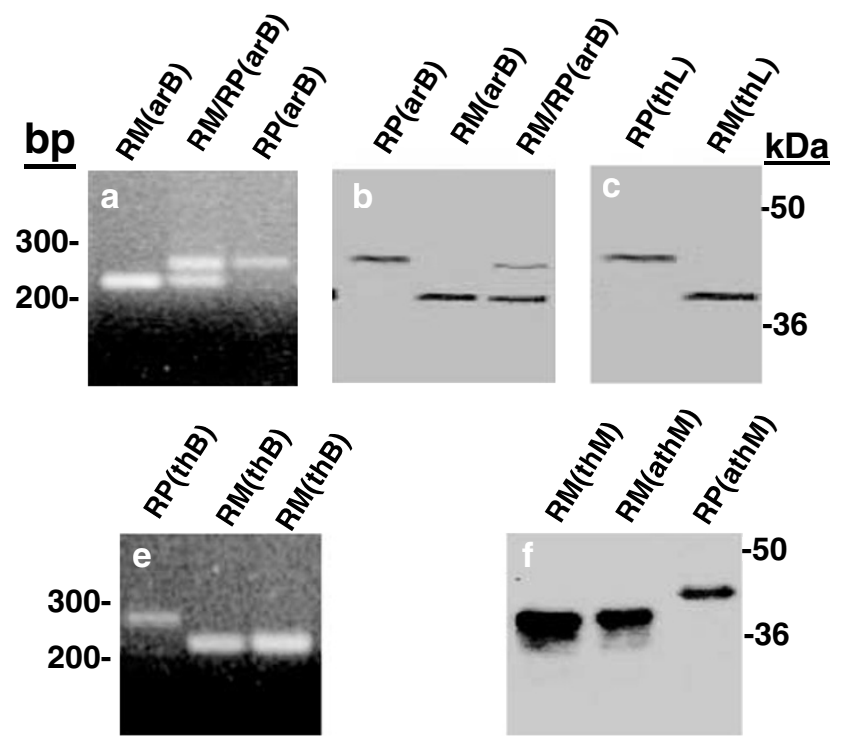

Figure 1 Molecular analysis of allelic genotypes at the VLP1-gene locus. (a) PCR analysis of genomic DNA from the sexual strain from Southern France, using primers flanking the site of the repeat sequence in the VLP1-coding region. Diagnostic DNA bands from homozygous and heterozygous VLP1 alleles are shown. (b) Western blot of protein extracts from the sexual strain from Southern France, using antibodies against a recombinant protein from the VLP1-gene (Beck et al, 1999). Diagnostic VLP1-protein bands from homozygous and heterozygous VLP1 alleles are shown. (c) Western blot of protein extracts from asexual wasps from the laboratory strain, using antibodies against a recombinant protein from the VLP1-gene (Beck et al, 1999). (d) PCR analysis of genomic DNA from the asexual strain from Southern France, using primers flanking the site of the repeat sequence in the VLP1-coding region. Diagnostic DNA bands from one RP-individual and two RM-individuals are shown. (e) Western blot of protein extracts from the asexual strain from Southern France, using antibodies against a recombinant protein from the VLP1-gene (Beck et al, 1999). Diagnostic VLP1-protein bands from two RM-individuals and one RP-individual are shown.

\section{The calyx phenotype}

The observed phenotypic difference in RP and RM calyx tissues is not fully penetrant and becomes evident when females are kept for several days, allowing the passage of eggs into the oviduct (Beck et al, 1999, 2001). To examine the phenotype of the homozygous RM or RP wasps, each female was kept for 6 days on honey-water without hosts, and the number of eggs inside the calyx and oviduct determined by dissections.

To determine the phenotype of heterozygous females, offspring from a genetic crosses between homozygous males and virgin females were kept for 6 days and egg numbers in the calyx and oviduct were examined as for homozygous females. More than 160 ovaries were recorded for each experiment and numbers analysed statistically using the JMPIN statistical package V3.2.1 (SAS Institute Inc., 1997).

\section{Reproductive success}

To assess the reproductive success of each wasp line under conditions that favour superparasitism, wasps from one line were exposed to hosts in the presence or absence of wasps from the other line. At $24 \mathrm{~h}$ before the start of an experimental trial, parasitoids and host patches were prepared. A total of 20 final instar E. kuehniella caterpillars were placed into a box together with some Ephestia diet. Freshly emerged wasps were isolated from homozygous RP and RM cultures and put into new culture vessels containing honey solution. On the day of the experiment, two healthy looking wasps were placed together with 20 caterpillars into a culture vessel $(6 \mathrm{~cm}$ in diameter, $9 \mathrm{~cm}$ high) and allowed to parasitise the hosts for either long or short time periods. In contrast to laboratory strains, which deposited eggs quickly after being together with caterpillars (Beck et al, 2001), it was observed that females collected from the field (both from Mildura and Southern France) required more time to oviposit. To differentiate between short and long periods of oviposition, 1 and 4 days were chosen as time periods.

Three different experimental conditions were examined. The RM(arB)-culture vessels contained two homozygous RM-wasps, the $\mathrm{RP}(\operatorname{arB})$-culture vessels two homozygous RP-wasps, and the RM/RP(arB)-vessels one homozygous RP and one homozygous RM wasp. The number and genetic identity of offspring were recorded and the average number of offspring per individual wasp from the $\mathrm{RM}(\operatorname{arB})$ and $\mathrm{RP}(\operatorname{arB})$-culture vessels compared to the number of offspring of the individual $\mathrm{RM}(\mathrm{arB})$ and $\mathrm{RP}(\mathrm{arB})$-wasps from the mixed wasp culture vessels $\mathrm{RM} / \mathrm{RP}(\mathrm{arB})$. Although we have no evidence for differences between male and female offspring success, the sex ratio of the two homozygous populations was determined to rule out any bias based on male/female distributions in superparasitised hosts. Whereas the absolute number of offspring was different for the two homozygous populations, the male/female ratio was similar (RM(arB) sex ratio 0.591 and $R P(\operatorname{arB})$ sex ratio 0.616 ), which excludes sex ratio differences as a possible basis for observed differences in the reproductive success.

\section{Polymerase chain reactions (PCR)}

Genomic DNA from the abdomen of individual wasps was extracted. PCR was performed using two VLP1specific primers, which were used to identify the tandem repeated sequence within the VLP1 protein (Hellers et al, 1996; Figure 1).

\section{Results}

Similar patterns of morphological and functional differences between the RM and RP lines were displayed by all three field-derived strains. Further, the observed patterns of differences were qualitatively the same as those reported for the pair of laboratory lines (Beck et al, 1999, 2001). However, all RM-lines showed higher degrees of variability compared to RP lines.

\section{Calyx gland phenotype}

Compared to the RP-lines, the RM-lines displayed higher levels of necrotic calyx cells, reduced expression of calyx fluid proteins and the presence of conspicuous membranes surrounding VLPs inside the calyx lumen. The calyx tissues from the RM and RP-lines of the three strains also revealed staining patterns similar to those for the laboratory lines: Compared to RP-ovaries (Figure 2a), staining of RM-calyx tissues was less intense and the VLP1-staining confined to the calyx lumen (Figure 2b). In contrast, RP-ovaries had more intensely stained calyx fluid, which was free to move into adjacent ovarioles 

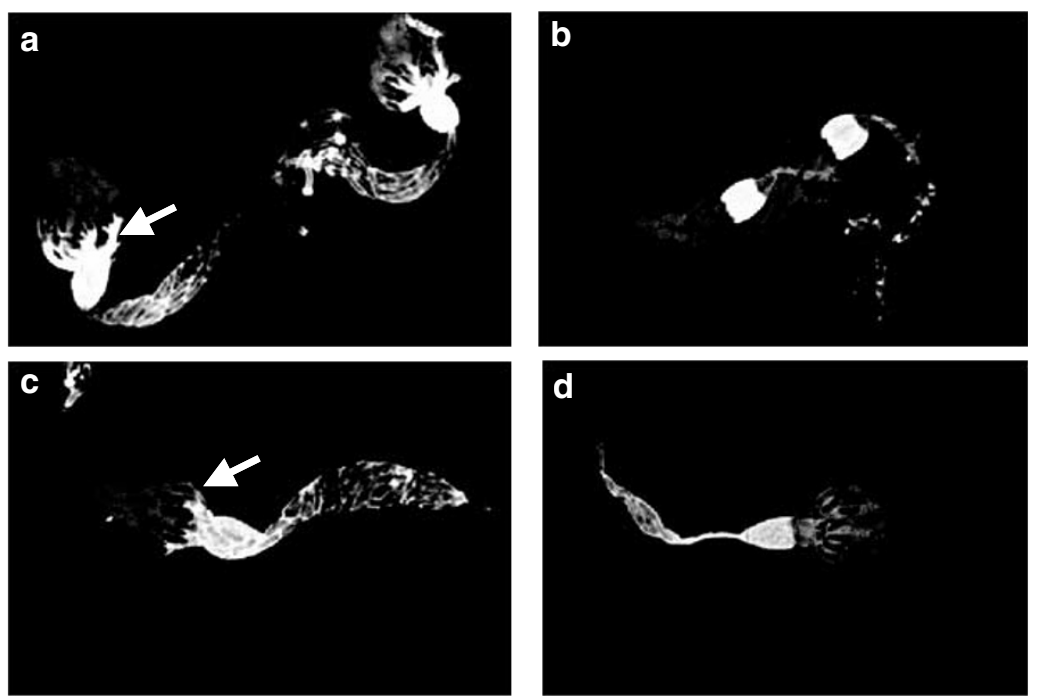

Figure 2 Antibody-stained ovaries from sexual and asexual females. Wholemounts of ovaries from asexual laboratory strains and sexual field-collected strains, using antibodies against a recombinant fusion protein from the VLP1-gene. Antibodies were visualised with FITCconjugated secondary antibodies under indirect UV-light: (a) Ovaries from an RP(thL) female, (b) ovaries from an RM(thL) female, (c) Ovaries from an RP(arB) female, (d) ovaries from an RM(arB) female. Note the staining of ovarioles adjacent to the calyx in RP-ovaries, due to the free movement of VLPs in the calyx lumen (arrows), whereas VLPs in RM-ovaries are surrounded by membranes and restricted to calyx tissues.

\section{Mean eggs in the calyx as a percentage of eggload for 7 day old wasps} from different strains

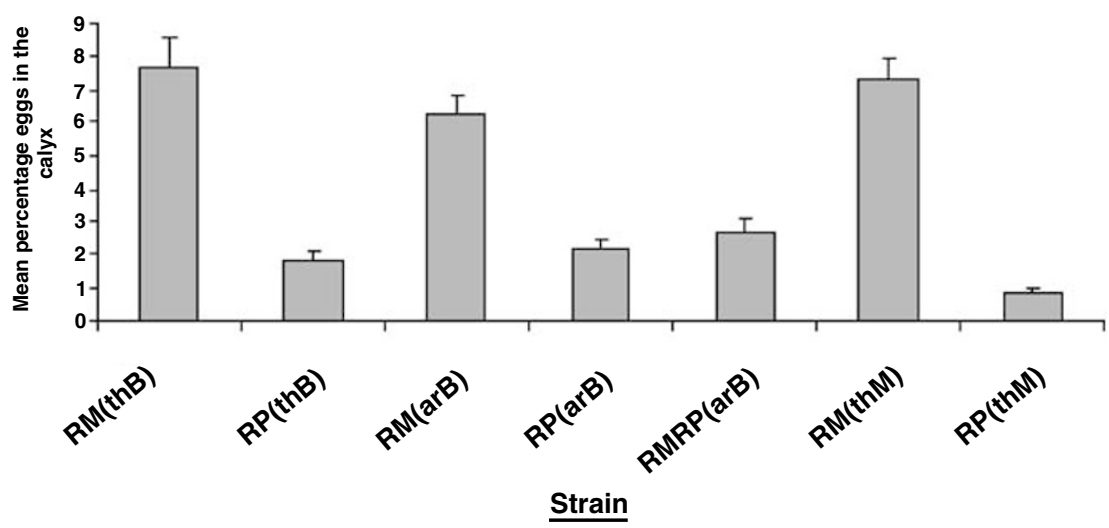

Figure 3 Distribution of egg numbers in calyx tissue of field-collected sexual and asexual females. The mean percentage of eggs found in the calyx tissues in ovaries from asexual females from Mont Boron (France) RP(thB) and RM(thB), from Mildura (Australia), RP(thM) and RM(thM), from homozygous and heterozygous sexual females from Mont Boron (France) RP(arB), RM(arB) and RP/RP(arB). Note that heterozygous ovaries resemble the RP-phenotype. The means segregated into two groups, with the means for the RM-ovaries significantly higher than the means for the RP- and heterozygous RM/RP(thB)-ovaries (Wilson-Tukey test, $P<0.0001$ ).

during dissection (Figure 2a, arrows). Likewise, staining in ovaries from homozygous sexual RP-females showed calyx fluid that was freely moving (Figure 2c), whereas in ovaries from sexual RM-females, staining of calyx fluid was restricted to calyx tissues (Figure 2d). This suggests that the differences in VLP1 protein amounts and distribution, observed in asexual laboratory lines, also exist in field populations and correlate with the two VLP1 genotypes. In other words, the cytological difference in asexual RP and RM-calyx tissues was also detected in the calyx of homozygous RP and RM females from sexual populations. This suggests that the calyx phenotype is genetically linked to the VLP1 locus.
Pleiotropic effects: egg distribution

In all three strains, the RM-line had more eggs inside the calyx tissues compared to the corresponding RP-line, while higher egg numbers were observed in the oviducts of RP-lines, compared to the corresponding RM-line. Thus, the phenotypic differences observed between the laboratory strains were also found in the field-collected strains (Figures 3 and 4).

When oviduct egg numbers were compared between the sexual and asexual strains, we noticed that egg numbers in asexual RP-oviducts were significantly higher than in sexual RP-oviducts. Likewise, egg 
Mean eggs in the oviduct of 7 day old wasps from related sexual and thelytokous strains

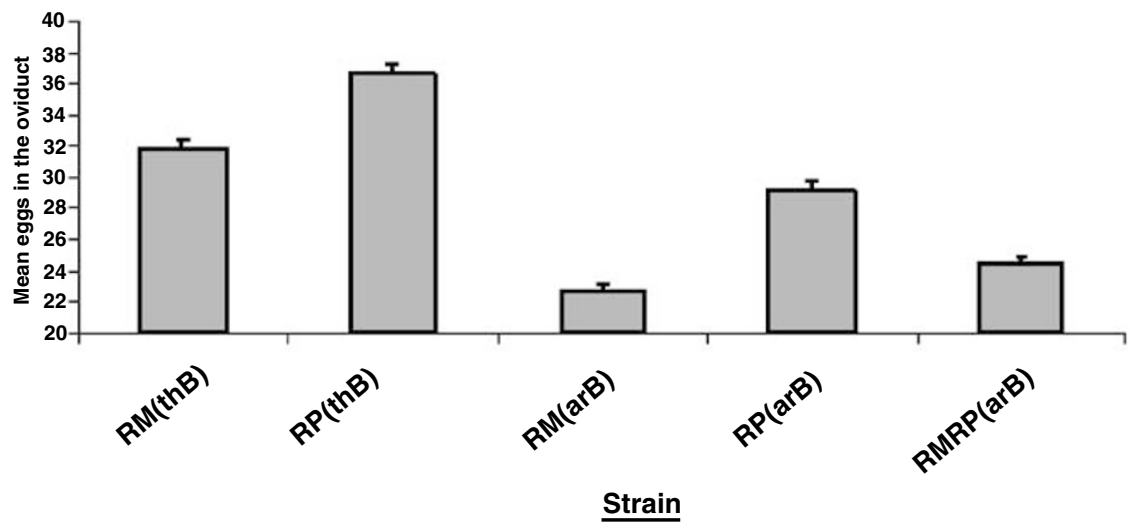

Figure 4 Distribution of egg numbers in oviducts of sexual and asexual females from Mont Boron (France). The mean number of eggs found in the oviducts from asexual females $\mathrm{RP}(\mathrm{thB})$ and from homozygous and heterozygous sexual females $\mathrm{RP}(\operatorname{arB}), \mathrm{RM}(\operatorname{arB}) \operatorname{and} \mathrm{RP} / \mathrm{RP}(\operatorname{arB})$. Note that heterozygous ovaries have an intermediate phenotype. All differences were significant (Wilson-Tukey test, $P<0.05$ in all cases).

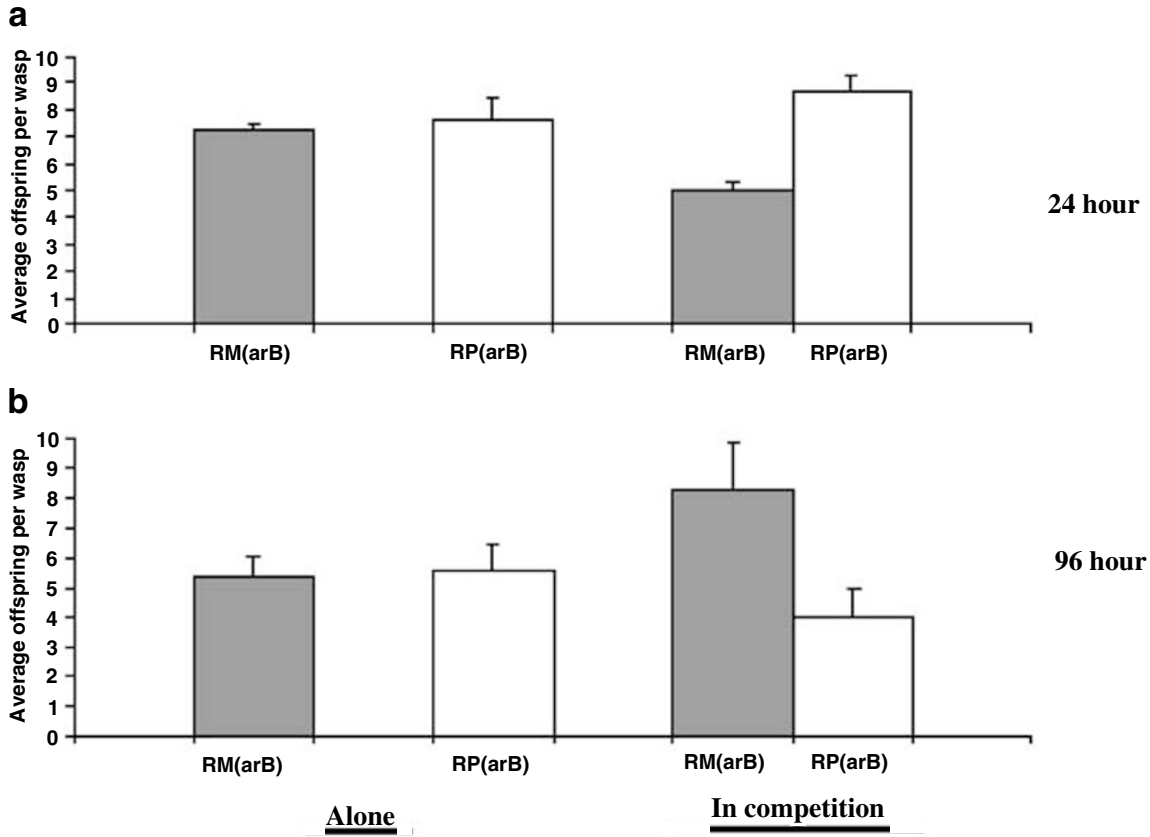

Figure 5 Reproductive success of sexual females under conditions where the two VLP1-genotypes compete inside host caterpillars. Pairs of homozygous VLP1-females were allowed to parasitise for 1 or 4 days. Reproductive success was monitored as a function of the average offspring per wasp. (a) Reproductive success after $24 \mathrm{~h}$ of egg deposition. Grey bars indicate average RM(arB) offspring and white bars average $\mathrm{RP}(\mathrm{arB})$ offspring. The grey column on the left shows average offspring from two RM(arB)-wasps, depositing eggs into the same hosts. The white column shows average offspring from two RP(arB)-wasps, depositing eggs into the same hosts. The two connected columns on the right show the average offspring from one of each $\mathrm{RP}(\mathrm{arB})$ - and $\mathrm{RM}(\operatorname{arB})$-wasps depositing eggs into the same hosts. RP(arB)-wasps produced significantly more offspring than $\mathrm{RM}(\mathrm{arB})$-wasps when competing for the same hosts for $24 \mathrm{~h}\left(\chi^{2}\right.$-test, $\left.P=0.0023\right)$. (b) Reproductive success after $96 \mathrm{~h}$ of egg deposition. Same column arrangements as in (a), where the grey column on the left represents two RM(arB)-wasps, the white column two RP(arB)-wasps, and the two columns on the right a mix of RP(arB)- and RM(arB)-wasps together. RP(arB)-wasps produced significantly fewer offspring than $\mathrm{RM}(\operatorname{arB})$-wasps when competing for the same hosts for $96 \mathrm{~h}\left(\chi^{2}\right.$-test, $\left.P=0.0006\right)$. There was no significant difference in the average number of offspring produced by the two genotypes when parasitising hosts separately, under either of the two time periods (grey and white individual columns in (a) and (b); however, both RP(arB)- and RM(arB)-wasps produced significantly fewer offspring when allowed to parasitise hosts for $96 \mathrm{~h}$ compared to $24 \mathrm{~h}$ ( $\chi^{2}$-test, $P=0.015$ and 0.0004 , respectively).

numbers in $\mathrm{RM}(\mathrm{thB})$-oviducts were higher than in RM(arB)-oviducts (Figure 4). This could indicate that an unlinked gene may interact with the VLP1-gene and that allelic combinations of the two genes are fixed in the asexual strains to produce the observed phenotypes (see Discussion).

\section{Reproductive success of sexual offspring}

When an RM(arB) and an RP(arB) female were allowed to deposit eggs in the same hosts for a limited period $(24 \mathrm{~h}) \mathrm{RP}(\mathrm{arB})$ females produced more offspring than $\mathrm{RM}(\operatorname{arB})$ females (Figure 5a, 24h), while when the time period was $96 \mathrm{~h}$ RM(arB) females were more successful 
(Figure 5b, 96h). However, when either two RM(arB) or two RP(arB) females were allowed to deposit eggs for the two time periods, there were no significant differences between the two genotypes in the number of offspring produced.

This suggests that the RM(arB) line is more successful when females from the two genotypes are allowed to compete for hosts over a relatively long period of time. This is consistent with our previous observations from laboratory populations that RP-females, with large egg reservoirs, deposit their eggs within a short period (Beck et al, 2001).

When either two RM(arB) or two RP(arB) females were given access to hosts, both genotypes produced fewer offspring when allowed to parasitise hosts for $96 \mathrm{~h}$ compared to $24 \mathrm{~h}$. A similar result was reported by Sirot (1996), with the rate of superparasitism success decreasing as the time interval between oviposition increased.

\section{Discussion}

An asexual laboratory strain of $V$. canescens was previously separated into two genetically different lines, which differ in ovarian morphology and reproductive strategies (Beck et al, 1999, 2001). The recent discovery of sexual and asexual $V$. canescens populations in Southern France (Malmberg et al, 2000; Schneider et al, 2002) enabled us to test the genetic basis of the observed differences between the two lines. The two questions of particular interest were whether the allelic VLP1-gene locus is linked to the phenotype described for the laboratory strain, and whether these phenotypic differences are also observed in field-collected wasp populations.

Since the RM and RP homozygous sexual lines were derived from a single population containing females heterozygous for the VLP1-gene, it is reasonable to assume that any genes not linked to the VLP1-gene locus would occur at similar frequencies in offspring from the two separated lines. When the offspring of genetic crosses of the two lines were analysed for the ovarian phenotype observed in the laboratory strain (Beck et al, 1999, 2001), the segregation of homozygous VLP1 offspring correlated with the ovarian phenotype, which suggests that the VLP1-gene is genetically linked to the phenotype. However, genetic linkage does not constitute proof that the allelic VLP1-gene is causing the phenotype. Further experiments, including molecular analysis of the VLP function and phenotypic rescue by genetic transformation experiments, would be required for genetic proof for the cause of the phenotype. Nevertheless, the genetic linkage of the VLP1-gene to the observed phenotype adds to already existing indirect evidence that the VLP1-gene is functionally related to the phenotype: Firstly, the gene is highly expressed in the calyx tissue, which is the primary focus of the phenotype (Beck et al, 1999). Secondly, the amount of secreted VLP1protein differs in the calyx lumen of the two strains (Figure 2). Thirdly, the predicted membrane-association of the VLP1-protein (Hellers et al, 1996) coincides with the appearance of conspicuous membranes in the calyx lumen of RM-females (Beck et al, 2001). Although the predicted PHGPX-domain in the VLP1-protein is probably not involved in reducing oxidized lipids (Hellers et al, 1996), the VLP1-protein may, nevertheless, facilitate the binding and removal of modified lipid moieties from the VLP-membrane (Li et al, unpublished data). Finally, genetic linkage of VLP1-alleles with the phenotype in all asexual populations examined, including those from Southern France and Australia, is an indication that the VLP1-gene locus may contribute to the phenotype.

However, the VLP1-gene may not be the only gene contributing to the phenotypic effects observed in the asexual lines. Although the two VLP1-alleles segregate with the relative differences in the ovary distribution of eggs, the absolute egg numbers differ in the corresponding asexual and sexual genotypes (Figure 4). This suggests that an additional unlinked gene may be involved in the transfer of eggs from the ovarioles into the oviduct. Further experiments are required to examine whether the phenotype observed in asexual strains is a result of a genotype fixation of a combination of two or more unlinked allelic genes.

The cytological changes in the calyx appear to result in several pleiotropic effects, which can be seen as physiological trade-off effects leading to two distinct reproductive strategies. The blockage of egg passages in the RM-calyx is associated with lower egg numbers found in the oviduct (Figure 5). The blockage of eggs in the calyx in turn affects egg maturation in adjacent ovarioles and may ultimately reduce the mobilization of fat reserves. Thus, a phenotypic description of the two genotypes, which includes the pleiotropic effects could entail that RM-females predominantly have lower egg numbers and higher fat reserves, whereas RP-females have higher egg numbers and lower fat reserves. An outcome of this phenotypic difference may be two distinct reproductive strategies, where one line (RP) is more effective in exploiting large clusters of hosts, whereas the other line (RM) is more effective when hosts are rare and more widely distributed. However, the relevance of the two observed phenotypes in the field remains to be examined.

A remarkable functional difference between the two phenotypes exists in interlarval competition in superparasitised hosts, where the two asexual laboratory lines have been shown to differ in reproductive success under interstrain competition compared to intrastrain competition (Beck et al, 1999). While the mechanism underlying these differences is not known, the observation that the two genotypes exist as balanced polymorphisms and show similar qualitative phenotypic difference in the two corresponding genotypes of field-collected sexual and asexual strains (Figure 5), suggests that the two coexisting reproductive strategies may be relevant in the field.

In summary, the observed morphological and functional differences in two laboratory lines of asexual parasitoids (Beck et al, 1999, 2001) can be observed in females from field-collected asexual VLP1 lines and in homozygous VLP1 genotypes of a sexual strain, suggestive that the VLP1 gene alteration is genetically linked to the phenotype.

\section{Acknowledgements}

We thank Markus Beck, Annette Reineke and Gerhard Kubach for help and suggestions during the experiments and Leo Beukeboom, Carlos Bernstein, Gerhard Driessen and Jacques van Alphen for continuous supply of sexual 
strains and helpful discussions. This work was supported by an ARC grant to OS and an Adelaide University Scholarship (OPRS) to DL.

\section{References}

Agoze ME, Drezen JM, Renault S, Periquet G (1994). Analysis of the reproductive potential of diploid males in the wasp Diadromus pulchellus (Hymenoptera: Ichneumonidae). Bull Entomol Res 84: 213-218.

Beck M, Reineke A, Lorenz H, Theopold U, Schmidt O (2001). Two distinct reproductive strategies are correlated with an ovarian phenotype in co-existing parthenogenetic strains of a parasitic wasp. J Insect Physiol 47: 1189-1195.

Beck M, Siekmann G, Li D M, Theopold U, Schmidt O (1999). A maternal gene mutation correlates with an ovary phenotype in a parthenogenetic wasp population. Insect Biochem Mol Biol 29: 453-460.

Beukeboom LW (2001). Single-locus complementary sex determination in the ichneumonid Venturia canescens (Gravenhorst) (Hymenoptera). Neth I Zool 51: 1-15.

Fisher RC (1961). A study in insect multiparasitism. II. The mechanism and control of competition for possession of the host. J Exp Biol 38: 605-628.

Fisher RC (1963). Oxygen requirements and the physiological suppression of supernumerary insect parasitoids. J Exp Biol 40: $531-540$.

Hellers M, Beck M, Theopold U, Kamei M, Schmidt O (1996). Multiple alleles encoding a virus-like particle protein in the ichneumonid endoparasitoid Venturia canescens. Insect Mol Biol 5: 239-249.

Hubbard SF, Harvey IF, Fletcher JP (1999). Avoidance of superparasitism: a matter of learning? Animal Behav 57: 1193-1197.
Malmberg T, Beukeboom LW, Driessen G, Van Alphen JJM (2000). Distribution of a VLP-protein in sexual and asexual Venturia canescens populations (Hymenoptera). Proc Exp Appl Entomol Entomol Soc 11: 89-93.

Marris GC, Casperd J (1996). The relationship between conspecific superparasitism and the outcome of in vitro contests staged between different larval instars of the solitary endoparasitoid Venturia canescens. Behav Ecol Sociobiol 39: 61-69.

Salt G (1934). Experimental studies in insect parasitism. II Superparasitism. Proc $R$ Soc (London) B 114: 455-476.

Salt G (1961). Competition among insect parasitoids. Mechanisms in biological competition. Symp Soc Exp Biol 15: 96-119.

Schmidt O, Theopold U, Strand MR (2001). Innate immunity and its evasion and suppression by hymenopteran endoparasitoids. BioEssays 23: 344-351.

Schneider MV, Beukeboom LW, Driessen G, Lapchin L, Bernstein C, Alphen JJM (2002). Geographical distribution and genetic relatedness of sympatrical thelytokous and arrhenotokous populations of the parasitoid Venturia canescens (Hymenoptera). J Environ Biol 15: 191-200.

Sirot E (1996). The pay-off from superparasitism in the solitary parasitoid Venturia canescens. Ecol Entomol 21: 305-307.

Stouthammer R, Luck RF, Werren JH (1992) Genetics of sex determination and the improvement of biological control using parasitoids. Ann Entomol Soc Am 21: 427-435.

Trudeau D, Gordon DM (1989). Factors determining the functional response of the parasitoid Venturia canescens. Entomol Exp Appl 50: 3-6.

Van Alphen JJM, Visser ME (1990). Superparasitism as an adaptive strategy for insect parasitoids. Annu Rev Entomol 35: 59-79.

Visser ME, Luyckx B, Nell HW, Boskamp GJF (1992). Adaptive superparasitism in solitary parasitoids: marking of parasitized hosts in relation to the pay-off from superparasitism. Ecol Entomol 17: 76-82. 